\title{
Development of a tool to measure the clinical response to biologic therapy in uncontrolled severe asthma: the FEOS score.
}

\author{
Luis Perez de Llano ${ }^{1}$, Ignacio Davila ${ }^{2,3}$, Eva Martinez-Moragon ${ }^{4}$, Javier \\ Domínguez-Ortega $^{5}$, Carlos Almonacid ${ }^{6}$, Carlos Colás ${ }^{7}$, Juan Luis García-Rivero ${ }^{8}$, Loreto \\ Carmona $^{9}$, María Jesús García de Yébenes $^{10}$, and Borja G Cosio ${ }^{11}$ \\ ${ }^{1}$ Hospital Universitario Lucus Augusti \\ ${ }^{2}$ University of Salamanca \\ ${ }^{3}$ University Hospital \\ ${ }^{4}$ University Hospital Dr Peset, Valencia \\ ${ }^{5}$ Hospital La Paz Institute for Health Research (IdiPAZ) \\ ${ }^{6}$ Hospital Universitario Ramón y Cajal Servicio de Neumología \\ ${ }^{7}$ Instituto de Investigacion Sanitaria de Aragon-Hospital Clinico \\ ${ }^{8}$ Hospital Comarcal de Laredo \\ ${ }^{9}$ Instituto de Salud Musculoesquelética \\ ${ }^{10}$ Instituto de Salud Musculoesquelética. \\ ${ }^{11}$ Hospital Son Espases, Palma de Mallorca; CIBER Enfermedades Respiratorias \\ (CIBERES), Spain.
}

September 16, 2020

\begin{abstract}
Background: There is a lack of tools to holistically quantify the response to monoclonal antibodies (mAbs) in severe uncontrolled asthma (SUA) patients. The aim of this study was to develop a valid score to assist specialists in this clinical context. Methods: The score was developed in 4 subsequent phases: (1) elaboration of the theoretical model of the construct intended to be measured (response to mAbs); (2) definition and selection of items and measurement instruments by Delphi survey; (3) weight assignment of the selected items by multicriteria decision analysis (MCDA) using the Potentially All Pairwise RanKings of all possible Alternatives (PAPRIKA) methodology via the 1000Minds software; and (4) face validity assessment of the obtained score. Results: Four core items, with different levels of response for each of them, were selected: "severe exacerbations", "oral corticosteroid use", "symptoms" (evaluated by Asthma Control Test: ACT) and "bronchial obstruction" (assessed by FEV1 \% theoretical). "Severe exacerbations" and "oral corticosteroid maintenance dose" were weighted most heavily $(38 \%$ each), followed by "symptoms" (13\%) and "FEV1" (11\%). Higher scores in the weighted system indicate better response and the range of responses runs from 0 (worsening) to 100 (best possible response). Face validity was high (intraclass correlation coefficient: 0.86). Conclusions: The FEOS score (FEV1, Exacerbations, Oral corticosteroids, Symptoms) allows clinicians to quantify response in SUA patients who are being treated with mAbs.
\end{abstract}

\section{Hosted file}

full manuscript.doc available at https://authorea.com/users/359576/articles/481462development-of-a-tool-to-measure-the-clinical-response-to-biologic-therapy-inuncontrolled-severe-asthma-the-feos-score 